\title{
Halo Nuclei in a three-body model and universal characteristics of low-energy few-body systems
}

\author{
Lauro Tomio*, M. T. Yamashita*, T. Frederico $^{\dagger}$ and A. Delfino** \\ *Instituto de Física Teórica, Universidade Estadual Paulista, 01140-070, São Paulo, SP, Brazil. \\ ${ }^{\dagger}$ Instituto Tecnológico de Aeronáutica, CTA, 12228-900, São José dos Campos, SP, Brazil. \\ **Instituto de Física, Universidade Federal Fluminense, 24210-900, Niterói, RJ, Brazil.
}

\begin{abstract}
Within general characteristics of low-energy few-body systems, we revise some wellknown correlations found in nuclear physics, and the properties of low-mass halo nuclei in a threebody neutron-neutron-core model. In this context, near the critical conditions for the occurrence of an Efimov state, we report some results obtained for the neutron $-{ }^{19} \mathrm{C}$ elastic scattering.
\end{abstract}

Keywords: halo nuclei, Few body systems, three-body, bound-states

PACS: 21.10.Dr, 21.10.Gv, 21.45.-v, 03.65.Ge

\section{INTRODUCTION}

In this report, we first review some well-known few-body correlations, discovered in the nuclear physics context. Actually, this is quite relevant in view of recent experiments in cold atom laboratories [1]-[4], which are claiming to have observed manifestations of some universal aspects of few-body physics [5]-[7]. One should also realize that several low-energy few-body correlations, verified in few-nucleon systems, can be more deeply studied in atomic laboratories, considering the actual possibilities in varying the two-body interaction by using Feshbach resonance techniques [8]. Few-body correlations are also shown to be important to study properties of low-mass exotic nuclei, with neutron halos, which can be described as neutron-neutron-core $(n-n-c)$ three-body systems [9]-[15]. In view of that, we will also discuss universal aspects and scaling behavior of low-energy three-body systems, considering non-identical two kind of particles. The approach is further illustrated by considering the halo nucleus carbon- $20\left({ }^{20} \mathrm{C}\right)$ in a three-body $n-n-{ }^{18} \mathrm{C}$ model. After some comments on the several studies considering the neutron-deuteron system, we also report scattering results for the $n-{ }^{19} \mathrm{C}$ system in an effective range analysis, which is similar to previous analysis done for the neutrondeuteron case. In the last section we present the main conclusions and perspectives.

\section{CORRELATIONS IN FEW-BODY NUCLEAR PHYSICS}

Thomas collapse - In nuclear physics, one of the oldest discoveries, recognized as a proof of the finite range of nuclear forces (See Sec. IV of Bethe and Bacher [16]), was that the triton binding energy will collapse to infinite if the range of the two-body interaction goes to zero for a fixed two-body binding [17]. This effect was also referred as Thomas's theorem [18], considering that Thomas was able to conclude that the range

CP1265, VIII Latin American Symposium on Nuclear Physics and Applications edited by R. Alcaron, H. F. Arellano, P. L. Cole, and A. J. Kreiner

(C) 2010 American Institute of Physics 978-0-7354-0814-2/10/\$30.00 
of the nuclear forces could not be less than $1.10^{-13} \mathrm{~cm}$ [19]). The Thomas's collapse has been discussed in many other subsequent works, in the context of three-nucleon systems [20]-[22] as well as when discussing more general low-energy few-body properties and correlations [23]-[30]. In the study of nuclear forces, several other correlations among observables have been found in the study of few-nucleon interactions $[31,32]$ and nuclear matter [33]. Later on, the Thomas effect was shown [24] to be closely related to the Efimov effect [23], which was discovered in 1970.

Efimov effect - An apparently counter-intuitive effect, verified by Efimov [23], refers to the infinite number of $s$-wave three-body energy levels, which appear when a twobody state is exactly at the dissociation threshold. This effect was shown to appear as a consequence of the same singularity structure of the kernel of the nonrelativistic scattering integral equation of a three-body system in the $s$-wave, responsible for the Thomas collapse [24], such that it is related to the Thomas effect by a scaling transformation. It was also verified that both, Thomas and Efimov effects, occur in three dimensions but not in one or two dimensions. More precisely, as demonstrated by Jensen, Riisager and Fedorov [29], the Thomas and Efimov effects can only occur for dimensions between 2.3 and 3.8. A simple variational proof of the Thomas effect, provided in Ref. [26], shows clearly why it is related to the Efimov effect. The relation of both effects was also recognized in some other recent works, such as in Refs. [7, 34]. The experimental study of Efimov states became accessible in ultra-cold atom laboratories, in view of the possibilities to alter the two-body scattering length, in a wide range by using Feshbach resonance techniques [8]. In 2006, it was reported Efimov states in scattering using caesium atoms at 10 nanokelvin $[2,7]$. More recent reports of Efimov spectrum in ultracold atoms are given in Refs. [4].

Phillips line - One of the first general correlations among low-energy observables, found by Phillips [31] in the nuclear force investigations was a consistent linear correlation between the triton binding energy and the doublet nucleon-deuteron scattering length. This correlation is shown to be due to the fact that the binding energies of both the triton and the deuteron, as well as the energy eigenvalue of the two-nucleon singlet virtual state, are all small on the energy scale of nuclear forces [35]. This correlation was followed by several studies on the trinucleon observables [36]-[40]. In Ref. [40], the trinucleon system was considered in a two-body model with Coulomb effect on bound and scattering states, leading to an effective nucleon-deuteron interaction in order to explain most of these properties. As observed, the correlation of triton binding with the nucleon-deuteron scattering length can essentially be explained in a two-body model, by using the $N / D$ approach [37], or in a three-body model with zero-range nucleonnucleon interaction. With the conjecture that the effective interaction is dominated by a truncated $r^{-2}$ interaction at large distances, it was demonstrated that the trinucleon low-energy properties can be explained by varying only the short-range part of this effective interaction, keeping the long-range behavior unchanged. The parameters are evaluated by requiring that the effective interaction produce the experimental values of the neutron-deuteron $(n-d)$ scattering length, ${ }^{2} a_{n d}$ and the binding energies, ${ }^{3} \mathrm{H}$ and ${ }^{3} \mathrm{He}$, where for the ${ }^{3} \mathrm{He}$ case it was added the Coulomb interaction. Next, the Phillips plot was easily reproduced by varying the short-range part of the effective interaction. This model implies in ${ }^{2} a_{p d}(\sim 0.15 \pm 0.1 \mathrm{fm})$, consistent with the analysis of Refs. [38, 39]. 
Tjon line - Relating three and four-body observables, Tjon [32] observed another interesting correlation, such that the ${ }^{4} \mathrm{He}$ binding energy can be determined by the triton binding. This correlation was also studied by Perne and Kröger [41] and by Noyes using nonrelativistic equations for three and four particle systems, within a zero-range scattering [42]. The observed correlation implies that, within the renormalization group approach [43], there is no need of a new scale to describe the four nucleon system. However, it has been argued that such result may be particular to the nuclear potential models used in the calculations, with their strong short-range repulsive interaction [44]. In a more general context, a possible four-body scale independent of the three-body one has being discussed in Refs. [45, 44]. As argued in [44] that it may be not valid near a Feshbach resonance in atomic systems, some experimental studies are being carried out in cold-atom laboratories [46], with actual analysis supporting the prediction that the universal tetramer states are in close connection with Efimov trimer [45].

Coester band - Another interesting correlation, between the nuclear matter binding energy per nucleon and the Fermi momentum, was verified by Coester, Day and Goodman [33], when studing the nuclear matter saturation curves for reasonable equivalent two-body potentials, following the observation that for any given two-body Hamiltonian there exists a large class of unitarily equivalent Hamiltonians that lead to the same scattering phase shifts at all energies. This Coester band was recently studied in Ref. [47], in a search for possible generalizations of the Tjon line to the many-nucleon context, from light nuclei to nuclear matter. As the nuclear matter saturates due to the composed repulsive and attractive short-ranged two-nucleon potential, it may also seen as a typical low-energy problem. So, it is natural to search for possible connections between the proper few-body scales with those of many-body problem. It was pointed out evidences for scaling between light nuclei binding energies and the triton, and argued that the saturation energy and density of nuclear matter are correlated to the triton binding energy, $B_{t}$. The available systematic nuclear matter calculations indicates a possible band structure representing these correlations. It was found that such Coester band can be seen as robustly represented by the scaling of nuclear matter properties with the triton binding.

\section{UNIVERSALITY AND SCALING LIMIT}

In order to understand and classify low-energy few-body correlations, the concepts of universality, scaling limit, or limit cycle, together with renormalization procedures, have been considered in many recent works. The relevance of such studies can be appreciated in view of the recent experiments [1]-[4] that are confirming universal properties coming from the Efimov physics [23] occurring for large two-body scattering lengths $[28,29,5,48]$. Near a Feshbach resonance the two-atom scattering length, $a$, can vary from very large negative values to positive values, allowing virtual or weaklybound dimers. The scattering length is large in respect to the atom-atom interaction range $\left(r_{0}\right)$, driving to the use of concepts developed for short range interactions and halo states [29]. In the limit of large $a$, the interaction can be taken as of zero range [49]. The appearance of Thomas-Efimov states in three-boson systems is controlled by the ratio $r_{0} /|a| \rightarrow 0$. In this exact limit, it is observed an infinite sequence of three-body bound 

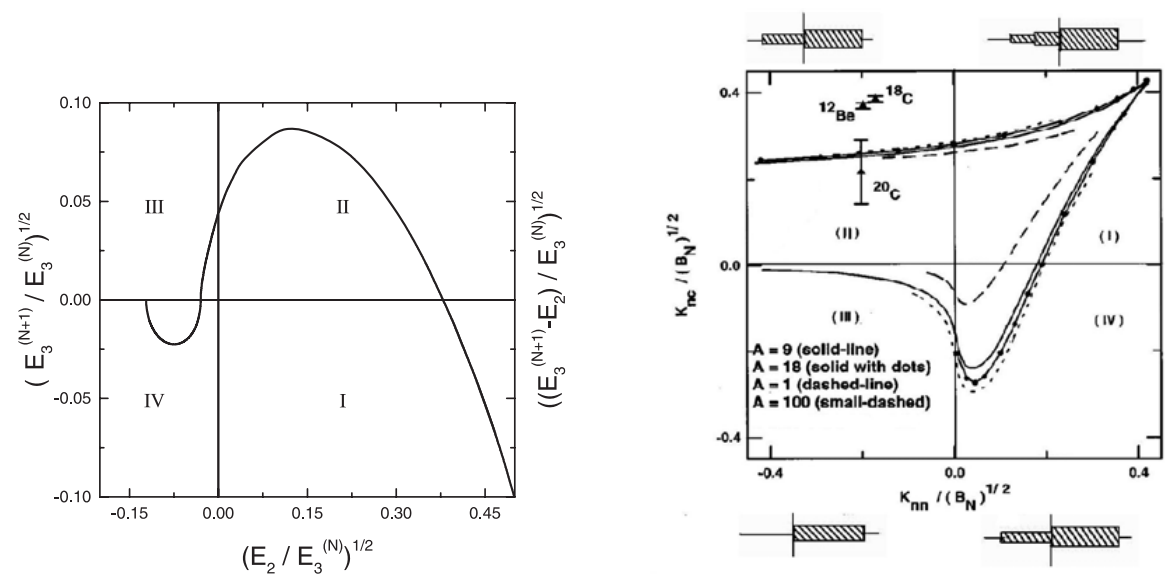

FIGURE 1. In the lhs figure, for identical three-body system, we have the ratio of the $(\mathrm{N}+1)^{\text {th }}$ trimer energy (bound, virtual, or resonant state), $E_{3}^{(N+1)}$, with respect to the threshold, two-body binding energy $E_{2}$. All quantities in units of the $\mathrm{N}^{\text {th }}$ trimer binding energy, $E_{3}^{(N)}$. For bound dimers, we have the regions I (virtual trimer) and II (bound trimer); with virtual dimers in regions III (virtual trimer) and IV (resonant trimer). The right vertical label is for regions I and II, with the left one for regions III and IV. In the rhs figure, we consider a three-body $n n c$ system, with mass ratio $A \equiv m_{c} / m_{n}$. A parametric region is defined by $K_{n n} / \sqrt{B_{N}}$ and $K_{n c} / \sqrt{B_{N}}$ (1). At the boundaries, the $(N+1)$ th Efimov state is zero (see Ref. [11]). The analytical two- and three-body cut structures are represented near the corresponding region.

states [11, 50], identified [51] with an underlying renormalization group limit cycle [43]. Such sequence of Efimov states, appearing as the two-body subsystem energy is reduced to zero, was presented in [50] as a scaling function when considering the interaction of three particles system $\alpha-\alpha-\beta$, with the corresponding masses given by $M_{\alpha}$ and $M_{\beta}$ having the mass-ratio $A \equiv M_{\beta} / M_{\alpha}$. The scaling function is given by the ratio of two consecutive three-body energy states $B_{3}^{N}$ and $B_{3}^{N+1}$ (the ground-state correspond to $N=0)$ :

$$
\frac{B_{3}^{N+1}}{B_{3}^{N}}=\mathscr{F}\left(\frac{K_{\alpha \alpha}}{\sqrt{B_{3}^{N}}}, \frac{K_{\alpha \beta}}{\sqrt{B_{3}^{N}}} ; A\right), \text { with }\left.\quad K_{\alpha \gamma}\right|_{\gamma \equiv \alpha, \beta} \equiv \frac{1}{a_{\alpha \gamma} \sqrt{2 \mu_{\alpha \gamma}}}= \pm \sqrt{B_{\alpha \gamma}}
$$

where, for the $(\alpha \gamma)$ system, $\mu_{\alpha \gamma}$ is the reduced mass, $a_{\alpha \gamma}$ is the scattering length, and $B_{\alpha \gamma}$ the energy for a bound (+) or virtual (-) two-body state. This scaling limit was first presented in Fig. 2 of Ref. [50], considering three identical particles, and compared with realistic model calculations for the ${ }^{4} \mathrm{He}$ trimer. In the lhs of Fig. (1), for three identical particles, we display Efimov states following a continuous transition when the two-body scattering length $a$ is varied. A virtual state emerges by the elastic scattering cut coming from the second Riemmann sheet becoming a bound state. Then, for a virtual two-body state $(a<0)$, a three-body bound state turns into a resonance. In the rhs of Fig. 1, for an $\alpha-\alpha-\beta$ system with $\alpha \equiv n$ and $\beta \equiv c$, it is shown the parametric region where at least one Efimov excited state can exist [11]. From the experimental data that were examined, only the carbon-20 was found with possibility to have at least one excited Efimov state. 
It is also shown in this figure the analytical cut structure of each four regions of the plane. The borromean case, where all two-body subsystems are unbound, is labelled as region (III). As discussed in Ref. [52] for the trajectories of excited three-body Efimov states, when at least one two-body subsystem is bound, the Efimov bound state turns to a virtual state when decreasing the corresponding scattering length. However, in the Borromean case, such three-body bound state turns to a resonance.

\section{HALO NUCLEI - EFIMOV STATES AND SCATTERING}

Apart from the actual interest brought by the possibilities in atomic laboratories, the investigations of Efimov states had also a renewed interest in the nuclear context, with the studies on the properties of exotic nuclei systems with two halo neutrons $(n-n)$ and a core $(c)$ [29]. In this respect, some of the most favorable systems for searching Efimov states in systems with two-neutron halos are light nuclei such as ${ }^{6} \mathrm{He},{ }^{11} \mathrm{Li},{ }^{14} \mathrm{Be}$, and carbon isotopes ${ }^{18,20,22} \mathrm{C}$. Among these systems, it was found that one of the possible candidates to present these states is the ${ }^{20} \mathrm{C}[11,12,29,53]$. Actually, it is also very promissing to consider the ${ }^{22} \mathrm{C}$ within a three-body $n-n-{ }^{20} \mathrm{C}$ model, in view of recent experimental properties obtained for this carbon isotope [15]. The sizes of such systems was studied in a zero-range renormalization approach in Ref. [14], within a general classification scheme of A-A-B systems, which was also extended to weakly bound molecules as well. By analyzing the dimensionless rootmean-square distances $\sqrt{\left\langle r_{n A}^{2}\right\rangle E_{3}}$ and $\sqrt{\left\langle r_{n n}^{2}\right\rangle E_{3}}$ in terms of scaling functions depending on dimensionless products of scattering lengths and square-root of the neutron-neutron separation energy, the qualitative properties of the different possibilities of three-body systems are understood in terms of the effective attraction in the model: when a pair has a virtual state the effective interaction is weaker than when the pair is bound. Thus, a three-body system has to shrink to keep the binding energy unchanged if a pair which is bound turns to be virtual. Several examples illustrate this property, which show that dimensionless sizes, $\sqrt{\left\langle r_{n A}^{2}\right\rangle E_{3}}$ and $\sqrt{\left\langle r_{n n}^{2}\right\rangle E_{3}}$, increase from Borromean (unbound subsystems), to All-Bound configurations. And the size is expected to increase beyond limits when a nonvanishing three-body energy hits the scattering threshold, with the Borromean configuration being the only exception.

${ }^{20} \mathbf{C}$ Scattering properties - When studying the scattering properties of the halo nuclei ${ }^{20} \mathrm{C}$, in a three-body model $n-n-{ }^{18} \mathrm{C}$, a strong enhancement of the $n-{ }^{19} \mathrm{C}$ elastic cross section was verified [53]. Further analysis of this kind of systems are demanding in view of actual interest, not only in nuclear physics, but also in similar systems with mixture of two-atom species in cold-atom experiments [54].

For the analysis of the $n-n-{ }^{18} \mathrm{C}$, one should first note that the proximity of an Efimov state (bound or virtual) makes the elastic cross-section extremely sensitive to the corresponding $S$-matrix pole. Such system has only one unbound subsystem, similar to the neutron-deuteron $(n-d)$ case. By considering the analytical cut structure of $n-n-c$ systems, one can show that [52], when at least one two-body subsystem is bound, an Efimov bound state turns to a virtual state by decreasing one of the two- 
body scattering lengths. However, in the Borromean case, such three-body bound state turns to a resonance, as one can observe from the analytical cut structures of the four possible regions schematically represented in rhs of 1 . Following the studies on the trajectories of Efimov excited three-body states [52], the ${ }^{20} \mathrm{C}$ scattering properties were further explored in Ref. [55] by analysing the elastic scattering of a neutron on ${ }^{19} \mathrm{C}$ near the condition for an Efimov state of ${ }^{20} \mathrm{C}$. The $n-n-{ }^{18} \mathrm{C}$ halo system presents a virtual state that turns into an excited state when the ${ }^{19} \mathrm{C}$ binding is decreased [52]. As shown in [55], the real part of the elastic $s$-wave phase-shift $\left(\delta_{0}^{R}\right)$ presents a zero, or a pole in $k \cot \delta_{0}^{R}$, when the system has an Efimov excited or virtual state.

In view of the structure of the scattering $S$-matrix for the $n-{ }^{19} \mathrm{C}$ system, one should recall the long-time discussion on the $n-d$ elastic doublet state scattering $[56,57,58,59$, $60,61,62]$. The change in the off-shell behavior of the two-body potential, or three-body forces, modifies the corresponding phase-shift correlated to the triton binding in a way that the scattering length can vanish. In this case, $k \cot \delta_{0}$ has a pole at zero relative $n-d$ kinetic energy, pointed out in the analysis of the experimental data for the $n-d$ doublet $s$-wave phase-shifts [56, 57]. Van Oers and Seagrave [57] proposed to incorporate a pole in a phenomenological effective range formula used to fit the $k \cot \delta_{0}$ low-energy data for the $n-d s$-wave doublet state just below the elastic threshold. The effective range expansion has a form given by

$$
k \cot \delta_{0}=-A+B k^{2}-\frac{C}{1+D k^{2}},
$$

where $A, B, C$, and $D$ are fitted constants. The existence of the triton virtual state was found on the basis of the effective range expansion [60]. From the solution of a threebody model with separable two-body interactions, it was also suggested that the triton virtual state appears from an excited Efimov state moving to the non-physical energy sheet through the elastic cut [63]. The same reasoning can be applied to the case of an excited Efimov state of the $n-n-{ }^{18} \mathrm{C}$ system, where the pole of the S-matrix migrates to the second energy sheet through the elastic $n-{ }^{19} \mathrm{C}$ cut when the binding energy of the neutron in ${ }^{19} \mathrm{C}$ is increased. The physics related to the Efimov effect is also implying a zero in the atom-dimer scattering length, and consequently a pole in the effective range expansion at zero kinetic energy. A pole in the effective range expansion of the $n-{ }^{19} \mathrm{C}$ elastic phase-shift appears in a quite good qualitative agreement with the above analysis. In this case, the low-energy parametrization of the effective range expansion is given by:

$$
k \cot \delta_{0}^{R}=\frac{-a_{n-19}^{-1}+\beta E+\gamma E^{2}}{1-E / E_{0}},
$$

where $a_{n-19} C$ is the $n-{ }^{19} C$ scattering length, with $\beta$ and $\gamma$ the effective range parameters to be adjusted. $E_{0}$ is the position of the pole with respect to the threshold for elastic scattering. Numerical solutions for $\left(1-E / E_{0}\right) k \cot \delta_{0}^{R}$ as a function of the CM kinetic energy are presented in [55], for different values of $\left|E_{19} C\right|$ between 200 and $850 \mathrm{keV}$. The effective range parameters, obtained from a fit of (3), are given in a table of Ref. [55]. 


\section{SUMMARY WITH PERSPECTIVES}

In view of the actual rich perspectives opened by recent experimental researches with few-atom and molecular systems in ultracold laboratories, where the two-body interaction can be varied by using Feshbach resonance techniques, it became quite relevant to revise some previous correlations first verified in few-body nuclear physics. Actually, in atomic and molecular physics, such few-body correlations can be tested with different kind of particles, from Bosons to Fermions, and one can further study the extension of such correlations. Within the renormalization group approach, the observed correlation are governed by some physical scales. As one increases the number of interacting particles, one can also study the possible need of independent new scales to describe the corresponding observables. So, the present report started with a briefly review of some low-energy few-nucleon correlations quite well-known by the nuclear physicists, as the Thomas-Efimov effect, Phillips, Tjon and Coester lines. Universal aspects of low-energy few-body physics are discussed in this context, together with some recent related works.

Next, it was discussed light halo-nuclei properties, considering the sizes of such threebody systems, trajectories of Efimov states and scattering properties. For the scattering properties, the study was exemplified with the Carbon-20, in view of recent calculations done for the $n-{ }^{19} \mathrm{C}$ elastic cross section and phase-shift analysis. In this context, we recall some old studies on the neutron-deuteron properties.

\section{ACKNOWLEDGMENTS}

Work partially supported by the Brazilian agencies Fundação de Amparo à Pesquisa do Estado de São Paulo (FAPESP) and Conselho Nacional de Desenvolvimento Científico e Tenológico (CNPq).

\section{REFERENCES}

1. T. Weber et al., Phys. Rev, Lett. 91, 123201 (2003).

2. T. Kraemer et al., Nature 440, 315 (2006).

3. S. Knoop, et al., Nature Physics 5, 227 (2009).

4. M. Zaccanti, et al., Nature Physics 5, 586 (2009); S. E. Pollack, et al., Phy. Rev. Lett. 102, 090402 (2009); S. E. Pollack, D. Dries, and R. G. Hulet, Science 326, 1683 (2009); G. Barontini, et al., Phys. Rev. Lett. 103, 073202 (2009).

5. E. Braaten and H.-W. Hammer, Ann. of Phys. 322, 120 (2007).

6. I. Baccarelli, et al., Phys. Rep. 452, 1 (2007).

7. M. D. Lee, T Köhler and P. S. Julienne, Phys. Rev. A 76, 012720 (2007).

8. E. Timmermans, P. Tommasini, M. Hussein, A. Kerman, Phys. Rep. 315, 199 (1999).

9. D.V. Fedorov, A.S. Jensen, and K. Riisager, Phys. Rev. Lett. 73, 2817 (1994).

10. P. G. Hansen, A. S. Jensen, B. Jonson, Annu. Rev. Nucl. Part. Sci. 45, 591 (1995).

11. A. E. Amorim, T. Frederico, and L. Tomio, Phys. Rev. C56, R2378 (1997).

12. I. Mazumdar and V. S. Bhasin, Phys. Rev. C 56, R5 (1997).

13. A. Delfino, T. Frederico, M.S. Hussein, and L. Tomio, Phys. Rev. C 61, 051301 (2000).

14. M. T. Yamashita, T. Frederico, and L. Tomio, Nucl. Phys. A 735, 40 (2004).

15. K. Tanaka et al., Phys. Rev. Lett. 104, 062701 (2010).

16. H. A. Bethe and R. F. Bacher, Rev. Mod. Phys. 8, 82 (1936).

17. L. H. Thomas, Phys. Rev. 47, 903 (1935).

18. R. L. Hall, Proc. Phys. Soc. 91, 787 (1967).

19. R. L. Pease and H. Feshbach 88, 945 (1952).

20. L. M. Delves and A. C. Phillips, Rev. Mod. Phys. 41, 497 (1969); J. S. C. McKee, Rep. Prog. Phys. 33, 691 (1970); H. P. Noyes,Phys. Rev. Lett. 25, 321 (1970); B. F. Gibson and G. J. Stephenson, Phys. 
Rev. C 8, 1222 (1973).

21. J. E. Beam, Phys. Lett. 30B, 76 (1969); V. A. Alexxandrini and C. A. Garcia Canal, Nucl. Phys. A 133, 950 (1969); G. Pantis, H. Fiedeldey, and D. W. L. Sprung, Z. Phys. A 291, 367 (1979); G. Rupp, L. Streit, and J. A. Tjon, Phys. Rev. C 31, 2285 (1985).

22. F. Tabakin, Phys. Rev. 174, 1208 (1968).

23. V. Efimov, Phys. Lett. B 33, 563 (1970).

24. S. K. Adhikari, A. Delfino, T. Frederico, I. D. Goldman, and L. Tomio, Phys. Rev. A 37, 3666 (1988).

25. A. Delfino, S. K. Adhikari, and L. Tomio, Phys. Rev. C 46, 471 (1992).

26. F. A. B. Coutinho, J. F. Perez, and W. F. Wreszinski, J. Math. Phys. 36, 1625 (1995).

27. A. Delfino, T. Frederico, and L. Tomio, Few-Body Syst. 28, 259 (2000).

28. P. F. Bedaque and U. van Kolck, Ann. Rev. Nucl. Part. Sci. 52, 339 (2002).

29. A. S. Jensen, K. Riisager, D. V. Fedorov, and E. Garrido Rev. Mod. Phys. 76, 215 (2004).

30. E. Braaten and H.-W. Hammer, Phys. Rep. 428, 259 (2006).

31. A. C. Phillips, Nucl. Phys. A 107, 209 (1968); Rep. Prog. Phys. 40, 905 (1977).

32. J.A. Tjon, Phys. Lett. B 56, 217 (1975).

33. F. Coester, B. Day, and A. Goodman, Phys. Rev. C 1, 769 (1970) and Phys. Rev. C 5, 1135 (1972).

34. B.D. Esry and C.H. Greene, Nature 440, 289 (2006).

35. V. E. Efimov and Tkachenko, Phys. Lett. 157B, 108 (1985).

36. H. Zankel and L. Mathelitsch, Phys. Lett. 132B, 27 (1983); G.H. Berthold and H. Zankel, ibid. 162B, 208 (1985); G.H. Berthold and H. Zankel, Phys. Rev. C 34, 1203 (1986).

37. S.K. Adhikari and J.R.A. Torreão, Phys. Lett. 132B, 257 (1983).

38. J.L Friar, B.F. Gibson, G.L. Payne, and C.R. Chen, Phys. Rev. C 30, 1121 (1984).

39. C.R. Chen, G.L. Payne, J.L Friar, and B.F. Gibson, Phys. Rev. C 33, 401 (1986).

40. L. Tomio, A. Delfino, and S.K. Adhikari, Phys. Rev. C 35, 441 (1987).

41. R. Perne and H. Kröger, Phys. Rev. C 20, 340 (1979).

42. H. P. Noyes, Phys. Rev. C 26, 1858 (1982).

43. K.G. Wilson, Phys. Rev. D3, 1818 (1971).

44. M. T. Yamashita, L. Tomio, A. Delfino, and T. Frederico, Europhys. Lett. 75, 555 (2006).

45. L. Platter, H.-W. Hammer and U.-G. Meissner, Phys. Lett. B 607, 254 (2005); H.-W. Hammer and L. Platter, Eur. Phys. J. A 32, 113 (2007).

46. F. Ferlaino, et al., Phys. Rev. Lett. 102, 140401 (2009).

47. A. Delfino, T. Frederico, V.S. Timóteo, and L.Tomio, Phys. Lett. B 634, 185 (2006).

48. E. Epelbaum, H.-W. Hammer, and Ulf-G. Meissner, Rev. Mod. Phys. 81, 1773 (2009).

49. R. Jackiw, in M. A. B. Beg Memorial Volume, edited by A. Ali and P. Hoodbhoy (World Scientific, Singapore, 1991).

50. T. Frederico, L. Tomio, A. Delfino, A. E. A. Amorim, Phys. Rev. A60, R9 (1999).

51. R.F. Mohr, R.J. Furnstahl, H.-W. Hammer, R.J. Perry, and K.G. Wilson, Ann. Phys. 321, 225 (2006).

52. M. T. Yamashita, T. Frederico and L. Tomio, Phys. Lett. B 660,339 (2008); Phys. Rev. Lett. 99, 269201 (2007).

53. V. Arora, I. Mazumdar and V. S. Bhasin, Phys. Rev. C 69, 061301(R) (2004); I. Mazumdar, A.R.P. Rau, V.S. Bhasin, Phys. Rev. Lett. 97, 062503 (2006).

54. B. Marcelis, S.J.J.M.F.Kokkelmans, G.V. Shlyapnikov, and D.S. Petrov, Phys. Rev. A 77, 032707 (2008).

55. M. T. Yamashita, T. Frederico, and L. Tomio, Phys. Lett. B 670, 49 (2008).

56. L.M. Delves, Phys. Rev. 118, 1318 (1960).

57. W.T.H. van Oers and J.D. Seagrave, Phys. Lett. 24B, 562 (1967).

58. A. S. Reiner, Phys. Lett. 28B, 387 (1969).

59. J.S. Whiting and M.G. Fuda, Phys. Rev. C 14, 18 (1976).

60. B. A. Girard and M.G. Fuda, Phys. Rev. C 19, 579 (1979).

61. S. K. Adhikari and J. R. A. Torreão, Phys. Lett. 119B, 245 (1982); Phys. Lett. 132B 257 (1983); S. K. Adhikari, Phys. Rev. C 30, 31 (1984).

62. I. V. Simenog, A. I. Sitnichenko and D. V. Shapoval, Sov. J. Nucl. Phys. 45, 37 (1987).

63. S. K. Adhikari, A. C. Fonseca, and L. Tomio, Phys. Rev. C 26, 77 (1982); S. K. Adhikari and L. Tomio, Phys. Rev. C 26, 83 (1982); S. K. Adhikari, L. Tomio and A. C. Fonseca, Phys. Rev. C 27, $1826(1983)$. 
Copyright of AIP Conference Proceedings is the property of American Institute of Physics and its content may not be copied or emailed to multiple sites or posted to a listserv without the copyright holder's express written permission. However, users may print, download, or email articles for individual use. 